\title{
CONCENTRATION PHENOMENA IN NONLINEAR EIGENVALUE PROBLEMS WITH VARIABLE EXPONENTS AND SIGN-CHANGING POTENTIAL
}

\author{
By \\ Mihai MihăIlescu AND VicenţiU Rădulescu
}

\begin{abstract}
In this paper we establish the concentration of the spectrum in an unbounded interval for a class of eigenvalue problems involving variable growth conditions and a sign-changing potential. We also study the optimization problem for the particular eigenvalue given by the infimum of the associated Rayleigh quotient when the variable potential lies in a bounded, closed and convex subset of a certain variable exponent Lebesgue space.
\end{abstract}

\section{Introduction and preliminary results}

In this paper, we are concerned with the study of the eigenvalue problem

$$
\begin{cases}-\operatorname{div}\left(\left(|\nabla u|^{p_{1}(x)-2}+|\nabla u|^{p_{2}(x)-2}\right) \nabla u\right)+V(x)|u|^{m(x)-2} u & \\ =\lambda\left(|u|^{q_{1}(x)-2}+|u|^{q_{2}(x)-2}\right) u, & \text { for } x \in \Omega, \\ u=0, & \text { for } x \in \partial \Omega,\end{cases}
$$

where $\Omega \subset \mathbb{R}^{N}(N \geq 3)$ is a bounded domain with smooth boundary, $\lambda$ is a real number, $V$ is an indefinite sign-changing weight, and $p_{1}, p_{2}, q_{1}, q_{2}, m$ are continuous functions on $\bar{\Omega}$. The interest in analyzing this kind of problem is motivated by some recent advances in the study of eigenvalue problems involving variable exponent growth conditions. We refer especially to the results in $[13,14,18,19,20,22,24,25,26]$. Problem (1) can be placed in the context of the above results, since in the particular case when $q_{1}(x)=q_{2}(x)=q(x)$ for any $x \in \bar{\Omega}$ and $V \equiv 0$ in $\Omega$, it has been studied in [23]. More precisely, provided that $p_{1}, p_{2}$, $q: \bar{\Omega} \rightarrow(1, \infty)$ are continuous functions such that $q$ has a subcritical growth with respect to $p_{2}$ and

$$
1<p_{2}(x)<\min _{\bar{\Omega}} q \leq \max _{\bar{\Omega}} q<p_{1}(x), \quad \forall x \in \bar{\Omega},
$$


then the particular case of problem (1) described above was discussed in [23] and it has been shown that there exist two positive constants $\lambda_{0}$ and $\lambda_{1}$, with $\lambda_{0} \leq \lambda_{1}$, such that any $\lambda \in\left[\lambda_{1}, \infty\right)$ is an eigenvalue of the problem while any $\lambda \in\left(0, \lambda_{0}\right)$ is not an eigenvalue. The form of problem (1) becomes a natural extension of the problem studied in [23] with the presence of the potential $V$ in the left-hand side of the equation and by considering that in the right-hand side we have $q_{1} \neq q_{2}$ on $\bar{\Omega}$.

The study of problems of type (1) has a strong motivation and important research efforts have been made with the aim of understanding anisotropic phenomena described by nonhomogeneous differential operators. We recall that equations of this type can be regarded as models for phenomena arising in the study of electrorheological fluids (see Halsey [15], Diening [6], Ružička [28]), elasticity (see Zhikov [31]) or image processing and restoration (see Chen, Levine and Rao [5], Esedoglu and Osher [12]). A survey of the history of this research field with a comprehensive bibliography is provided by Diening, Hästö and Nekvinda [7].

In the present paper, we study problem (1) when $p_{1}, p_{2}, q_{1}, q_{2}, m: \bar{\Omega} \rightarrow(1, \infty)$ are continuous functions satisfying the following hypotheses:

(2)

$$
\max _{\bar{\Omega}} p_{2}<\min _{\bar{\Omega}} q_{2} \leq \max _{\bar{\Omega}} q_{2} \leq \min _{\bar{\Omega}} m \leq \max _{\bar{\Omega}} m \leq \min _{\bar{\Omega}} q_{1} \leq \max _{\bar{\Omega}} q_{1}<\min _{\bar{\Omega}} p_{1},
$$

$$
\max _{\bar{\Omega}} q_{1}<p_{2}^{\star}(x):= \begin{cases}\frac{N p_{2}(x)}{N-p_{2}(x)} & \text { if } p_{2}(x)<N \\ +\infty & \text { if } p_{2}(x) \geq N\end{cases}
$$

We assume that the potential $V: \Omega \rightarrow \mathbb{R}$ satisfies

$$
V \in L^{r(x)}(\Omega), \text { with } r \in C(\bar{\Omega}) \text { and } r(x)>\frac{N}{\min _{\bar{\Omega}} m} \quad \forall x \in \bar{\Omega} .
$$

Condition (2), which describes the competition between the growth rates involved in equation (1), represents the key of the present study since it establishes a balance between all the variable exponents involved in the problem. Such a balance is essential since our setting assumes a nonhomogeneous eigenvalue problem for which a minimization technique based on the Lagrange multiplier theorem cannot be applied in order to find (principal) eigenvalues (unlike the case offered by the homogeneous operators). Thus, in the case of nonlinear nonhomogeneous eigenvalue problems, the classical theory used in the homogeneous case does not work entirely, but some of its ideas can still be useful, and some particular results can still be obtained in some aspects, while in other aspects entirely new phenomena can occur. To focus on our case, condition (2) together with conditions (3) and (4) 
imply

$$
\lim _{\|u\|_{p_{1}(x) \rightarrow 0}} \frac{\int_{\Omega} \frac{1}{p_{1}(x)}|\nabla u|^{p_{1}(x)} d x+\int_{\Omega} \frac{1}{p_{2}(x)}|\nabla u|^{p_{2}(x)} d x+\int_{\Omega} \frac{V(x)}{m(x)}|u|^{m(x)} d x}{\int_{\Omega} \frac{1}{q_{1}(x)}|u|^{q_{1}(x)} d x+\int_{\Omega} \frac{1}{q_{2}(x)}|u|^{q_{2}(x)} d x}=\infty
$$

and

$$
\lim _{\|u\|_{p_{1}(x) \rightarrow \infty}} \frac{\int_{\Omega} \frac{1}{p_{1}(x)}|\nabla u|^{p_{1}(x)} d x+\int_{\Omega} \frac{1}{p_{2}(x)}|\nabla u|^{p_{2}(x)} d x+\int_{\Omega} \frac{V(x)}{m(x)}|u|^{m(x)} d x}{\int_{\Omega} \frac{1}{q_{1}(x)}|u|^{q_{1}(x)} d x+\int_{\Omega} \frac{1}{q_{2}(x)}|u|^{q_{2}(x)} d x}=\infty,
$$

where $\|\cdot\|_{p_{1}(x)}$ stands for the norm in the variable exponent Sobolev space $W_{0}^{1, p_{1}(x)}(\Omega)$. In other words, the absence of homogeneity is balanced by the behavior (actually, the blow-up) of the Rayleigh quotient associated to problem (1) in the origin and at infinity. The consequence of the above remarks is that the infimum of the Rayleigh quotient associated to problem (1) is a real number, i.e.,

$$
\begin{aligned}
& \inf _{u \in W_{0}^{1, p_{1}(x)}(\Omega) \backslash\{0\}} \frac{1}{\int_{\Omega} \frac{1}{q_{1}(x)}|u|^{q_{1}(x)} d x+\int_{\Omega} \frac{1}{q_{2}(x)}|u|^{q_{2}(x)} d x} \\
& \times\left(\int_{\Omega} \frac{1}{p_{1}(x)}|\nabla u|^{p_{1}(x)} d x+\int_{\Omega} \frac{1}{p_{2}(x)}|\nabla u|^{p_{2}(x)} d x+\int_{\Omega} \frac{V(x)}{m(x)}|u|^{m(x)} d x\right) \in \mathbb{R},
\end{aligned}
$$

and it is attained for a function $u_{0} \in W_{0}^{1, p_{1}(x)}(\Omega) \backslash\{0\}$. Moreover, the value in (5) represents an eigenvalue of problem (1) with the corresponding eigenfunction $u_{0}$. However, at this stage, we cannot say if the eigenvalue described above is the lowest eigenvalue of problem (1) or not, even if we are able to show that any small enough $\lambda$ is not an eigenvalue of (1). At the moment this remains an open question. On the other hand, we can prove that any $\lambda$ larger than the value given by relation (5) is also an eigenvalue of problem (1). Thus, we conclude that problem (1) possesses a continuous family of eigenvalues.

Related with the above ideas we also discuss the optimization of the eigenvalues described by relation (5) with respect to the potential $V$, provided that $V$ belongs to a bounded, closed and convex subset of $L^{r(x)}(\Omega)$ (where $r(x)$ is given by relation (4)). By optimization, we understand the existence of some potentials $V_{\star}$ and $V^{\star}$ such that the eigenvalue described in relation (5) is minimal or maximal with respect to the set where $V$ lies. The results that we obtain in the context of optimization of eigenvalues are motivated by the above advances in this field in the case of homogeneous (linear or nonlinear) eigenvalue problems. We refer mainly to the studies in Asbaugh-Harrell [2], Egnell [11] and Bonder-Del Pezzo [3], where different optimization problems of the principal eigenvalue of some homogeneous operators were studied. 
We start with some preliminary basic results on the theory of LebesgueSobolev spaces with variable exponent. For more details we refer to the book by Musielak [27] and the papers by Edmunds et al. [8, 9, 10], Kováčik and Rákosník [17], Mihăilescu and Rădulescu [21], and Samko and Vakulov [29].

Set

$$
C_{+}(\bar{\Omega})=\{h ; h \in C(\bar{\Omega}), h(x)>1 \text { for all } x \in \bar{\Omega}\} .
$$

For any $h \in C_{+}(\bar{\Omega})$, we define

$$
h^{+}=\sup _{x \in \Omega} h(x) \quad \text { and } \quad h^{-}=\inf _{x \in \Omega} h(x) .
$$

For any $p \in C_{+}(\bar{\Omega})$, we define the variable exponent Lebesgue space

$$
\begin{gathered}
L^{p(x)}(\Omega)=\{u ; u \text { is a measurable real-valued function such that } \\
\left.\qquad \int_{\Omega}|u(x)|^{p(x)} d x<\infty\right\} .
\end{gathered}
$$

We define on this space the Luxemburg norm by

$$
|u|_{p(x)}=\inf \left\{\mu>0 ; \int_{\Omega}\left|\frac{u(x)}{\mu}\right|^{p(x)} d x \leq 1\right\} .
$$

Let $L^{p^{\prime}(x)}(\Omega)$ denote the conjugate space of $L^{p(x)}(\Omega)$, where $1 / p(x)+1 / p^{\prime}(x)=1$. For any $u \in L^{p(x)}(\Omega)$ and $v \in L^{p^{\prime}(x)}(\Omega)$, the Hölder type inequality

$$
\left|\int_{\Omega} u v d x\right| \leq\left(\frac{1}{p^{-}}+\frac{1}{p^{\prime-}}\right)|u|_{p(x)}|v|_{p^{\prime}(x)}
$$

holds true.

An important role in manipulating the generalized Lebesgue-Sobolev spaces is played by the modular of the $L^{p(x)}(\Omega)$ space, which is the mapping $\rho_{p(x)}: L^{p(x)}(\Omega) \rightarrow \mathbb{R}$ defined by

$$
\rho_{p(x)}(u)=\int_{\Omega}|u|^{p(x)} d x .
$$

If $\left(u_{n}\right), u \in L^{p(x)}(\Omega)$, then the following relations hold:

$$
\begin{gathered}
|u|_{p(x)}>1 \quad \Rightarrow \quad|u|_{p(x)}^{p^{-}} \leq \rho_{p(x)}(u) \leq|u|_{p(x)}^{p^{+}}, \\
|u|_{p(x)}<1 \quad \Rightarrow \quad|u|_{p(x)}^{p^{+}} \leq \rho_{p(x)}(u) \leq|u|_{p(x)}^{p^{-}}, \\
\left|u_{n}-u\right|_{p(x)} \rightarrow 0 \quad \Leftrightarrow \quad \rho_{p(x)}\left(u_{n}-u\right) \rightarrow 0 .
\end{gathered}
$$


Next, we define $W_{0}^{1, p(x)}(\Omega)$ as the closure of $C_{0}^{\infty}(\Omega)$ under the norm

$$
\|u\|_{p(x)}=|\nabla u|_{p(x)} .
$$

The space $W_{0}^{1, p(x)}(\Omega)$ is a separable and reflexive Banach space. We note that if $s \in$ $C_{+}(\bar{\Omega})$ and $s(x)<p^{\star}(x)$ for all $x \in \bar{\Omega}$, then the embedding $W_{0}^{1, p(x)}(\Omega) \hookrightarrow L^{s(x)}(\Omega)$ is compact and continuous, where $p^{\star}(x)$ denotes the corresponding critical Sobolev exponent, that is,

or

$$
p^{\star}(x):=\frac{N p(x)}{N-p(x)} \quad \text { if } p(x)<N
$$

$$
p^{\star}(x)=+\infty \quad \text { if } p(x) \geq N .
$$

For applications of Sobolev spaces with variable exponent, we refer to Acerbi and Mingione [1], Chen, Levine and Rao [5], Diening [6], Halsey [15], Ružička [28], Zhikov [31] and Harjulehto et al. [16].

\section{The main results}

Since $p_{2}(x)<p_{1}(x)$ for any $x \in \bar{\Omega}$, it follows that $W_{0}^{1, p_{1}(x)}(\Omega)$ is continuously embedded in $W_{0}^{1, p_{2}(x)}(\Omega)$. Thus, a solution for a problem of type (1) will be sought in the variable exponent space $W_{0}^{1, p_{1}(x)}(\Omega)$.

We say that $\lambda \in \mathbb{R}$ is an eigenvalue of problem (1) if there exists

$$
u \in W_{0}^{1, p_{1}(x)}(\Omega) \backslash\{0\}
$$

such that

$$
\begin{aligned}
\int_{\Omega}\left(|\nabla u|^{p_{1}(x)-2}+|\nabla u|^{p_{2}(x)-2}\right) \nabla u \nabla v d x & +\int_{\Omega} V(x)|u|^{m(x)-2} u v \quad d x \\
& -\lambda \int_{\Omega}\left(|u|^{q_{1}(x)-2}+|u|^{q_{2}(x)-2}\right) u v \quad d x=0,
\end{aligned}
$$

for all $v \in W_{0}^{1, p_{1}(x)}(\Omega)$. We note that if $\lambda$ is an eigenvalue of problem (1), then the corresponding eigenfunction $u \in W_{0}^{1, p_{1}(x)}(\Omega) \backslash\{0\}$ is a weak solution of problem (1).

For each potential $V \in L^{r(x)}(\Omega)$, we define

$$
\begin{aligned}
E(V):= & \inf _{u \in W_{0}^{1, p_{1}(x)}(\Omega) \backslash\{0\}} \frac{1}{\int_{\Omega} \frac{1}{q_{1}(x)}|u|^{q_{1}(x)} d x+\int_{\Omega} \frac{1}{q_{2}(x)}|u|^{q_{2}(x)} d x} \\
& \times\left(\int_{\Omega} \frac{1}{p_{1}(x)}|\nabla u|^{p_{1}(x)} d x+\int_{\Omega} \frac{1}{p_{2}(x)}|\nabla u|^{p_{2}(x)} d x+\int_{\Omega} \frac{V(x)}{m(x)}|u|^{m(x)} d x\right)
\end{aligned}
$$


and

$$
F(V):=\inf _{u \in W_{0}^{1, p_{1}(x)}(\Omega) \backslash\{0\}} \frac{\int_{\Omega}|\nabla u|^{p_{1}(x)} d x+\int_{\Omega}|\nabla u|^{p_{2}(x)} d x+\int_{\Omega} V(x)|u|^{m(x)} d x}{\int_{\Omega}|u|^{q_{1}(x)} d x+\int_{\Omega}|u|^{q_{2}(x)} d x} .
$$

Thus, we can define a function $E: L^{r(x)}(\Omega) \rightarrow \mathbb{R}$.

The first result of this paper is given by the following theorem.

Theorem 1. Assume that conditions (2), (3) and (4) are fulfilled. Then $E(V)$ is an eigenvalue of problem (1). Moreover, there exists an eigenfunction $u \in$ $W_{0}^{1, p_{1}(x)}(\Omega) \backslash\{0\}$ corresponding to the eigenvalue $E(V)$ such that

$$
E(V)=\frac{\int_{\Omega} \frac{1}{p_{1}(x)}|\nabla u|^{p_{1}(x)} d x+\int_{\Omega} \frac{1}{p_{2}(x)}|\nabla u|^{p_{2}(x)} d x+\int_{\Omega} \frac{V(x)}{m(x)}|u|^{m(x)} d x}{\int_{\Omega} \frac{1}{q_{1}(x)}|u|^{q_{1}(x)} d x+\int_{\Omega} \frac{1}{q_{2}(x)}|u|^{q_{2}(x)} d x} .
$$

Furthermore, $F(V) \leq E(V)$, each $\lambda \in(E(V), \infty)$ is an eigenvalue of problem (1), while each $\lambda \in(-\infty, F(V))$ is not an eigenvalue of problem (1).

Next, we show that on each convex, bounded and closed subset of $L^{r(x)}(\Omega)$ the function $E$ defined above is bounded from below and attains its minimum. The result is the following.

Theorem 2. Assume that conditions (2), (3) and (4) are fulfilled. Assume that $S$ is a convex, bounded and closed subset of $L^{r(x)}(\Omega)$. Then there exists $V_{\star} \in S$ which minimizes $E(V)$ on $S$, i.e.,

$$
E\left(V_{\star}\right)=\inf _{V \in S} E(V) .
$$

Finally, we focus our attention on the particular case when the set $S$ from Theorem 2 is a ball in $L^{r(x)}(\Omega)$. Thus, we denote each closed ball centered in the origin of radius $R$ from $L^{r(x)}(\Omega)$ by $\bar{B}_{R}(0)$, i.e.,

$$
\bar{B}_{R}(0):=\left\{u \in L^{r(x)}(\Omega) ;|u|_{r(x)} \leq R\right\} .
$$

By Theorem 2, we can define the function $E_{\star}:[0, \infty) \rightarrow \mathbb{R}$ by

$$
E_{\star}(R)=\min _{V \in \bar{B}_{R}(0)} E(V) .
$$

Our result on the function $E_{\star}$ is given by the following theorem.

Theorem 3. (a) The function $E_{\star}$ is not constant and decreases monotonically.

(b) The function $E_{\star}$ is continuous. 
On the other hand, we point out that results similar to those of Theorems 2 and 3 can be obtained if we note that on each convex, bounded and closed subset of $L^{r(x)}(\Omega)$, the function $E$ defined in Theorem 1 is also bounded from above and attains its maximum. It is also easy to see that we can define a function $E^{\star}:[0, \infty) \rightarrow \mathbb{R}$ by

$$
E^{\star}(R)=\max _{V \in \bar{B}_{R}(0)} E(V),
$$

which has properties similar to $E_{\star}$.

\section{Proof of Theorem 1}

Let $X$ denote the generalized Sobolev space $W_{0}^{1, p_{1}(x)}(\Omega)$. We denote by $\|\cdot\|$ the norm on $W_{0}^{1, p_{1}(x)}(\Omega)$ and by $\|\cdot\|_{1}$ the norm on $W_{0}^{1, p_{2}(x)}(\Omega)$.

Define the functionals $J_{V}, I: X \rightarrow \mathbb{R}$ by

$$
\begin{gathered}
J_{V}(u)=\int_{\Omega} \frac{1}{p_{1}(x)}|\nabla u|^{p_{1}(x)} d x+\int_{\Omega} \frac{1}{p_{2}(x)}|\nabla u|^{p_{2}(x)} d x+\int_{\Omega} \frac{V(x)}{m(x)}|u|^{m(x)} d x, \\
I(u)=\int_{\Omega} \frac{1}{q_{1}(x)}|u|^{q_{1}(x)} d x+\int_{\Omega} \frac{1}{q_{2}(x)}|u|^{q_{2}(x)} d x .
\end{gathered}
$$

Note that for any $V$ satisfying condition (4), we have

$$
J_{V}(u)=J_{0}(u)+\int_{\Omega} \frac{V(x)}{m(x)}|u|^{m(x)} d x, \quad \forall u \in X,
$$

where $J_{0}$ is obtained in the case when $V=0$ in $\Omega$.

Standard arguments imply that $J_{V}, I \in C^{1}(X, \mathbb{R})$ and for all $u, v \in X$,

$$
\begin{aligned}
\left\langle J_{V}^{\prime}(u), v\right\rangle & =\int_{\Omega}\left(|\nabla u|^{p_{1}(x)-2}+|\nabla u|^{p_{2}(x)-2}\right) \nabla u \nabla v d x+\int_{\Omega} V(x)|u|^{m(x)-2} u v d x, \\
\left\langle I^{\prime}(u), v\right\rangle & =\int_{\Omega}|u|^{q_{1}(x)-2} u v d x+\int_{\Omega}|u|^{q_{2}(x)-2} u v d x .
\end{aligned}
$$

In order to prove Theorem 1, we first establish some auxiliary results.

Lemma 1. Assume conditions (2), (3) and (4) are fulfilled. Then for each $\epsilon>0$ there exists $C_{\epsilon}>0$ such that

$$
\begin{aligned}
\left.\left|\int_{\Omega} \frac{V(x)}{m(x)}\right| u\right|^{m(x)} d x \mid \leq & \epsilon \int_{\Omega}\left(\frac{1}{p_{1}(x)}|\nabla u|^{p_{1}(x)} d x+\frac{1}{p_{2}(x)}|\nabla u|^{p_{2}(x)}\right) d x \\
& +C_{\epsilon}|V|_{r(x)} \int_{\Omega}\left(|u|^{m^{-}}+|u|^{m^{+}}\right) d x,
\end{aligned}
$$

for all $u \in X$. 
Proof. First, we point out that since $r(x)>r^{-}$on $\bar{\Omega}$, it follows that $L^{r(x)}(\Omega) \subset$ $L^{r^{-}}(\Omega)$. On the other hand, since $r(x)>N / m^{-}$for each $x \in \bar{\Omega}$, it follows that $r^{-}>N / m^{-}$. Thus, we infer that $V \in L^{r^{-}}(\Omega)$ and $r^{-}>N / m^{-}$.

Now let $\epsilon>0$ be fixed. We claim that there exists $D_{\epsilon}>0$ such that

$$
\begin{aligned}
\int_{\Omega}|V(x)| \cdot|u|^{m^{-}} d x \leq \epsilon \int_{\Omega}|\nabla u|^{m^{-}} d x+D_{\epsilon}|V|_{r^{-}} \int_{\Omega}|u|^{m^{-}} & d x, \\
& \forall u \in W_{0}^{1, m^{-}}(\Omega) .
\end{aligned}
$$

In order to establish (10), we show first that for each $s \in\left(1, \frac{\mathrm{Nm}^{-}}{\mathrm{N}-\mathrm{m}^{-}}\right)$, there exists $D_{\epsilon}^{\prime}>0$ such that

$$
|v|_{s} \leq \epsilon|| \nabla v||_{m^{-}}+D_{\epsilon}^{\prime}|v|_{m^{-}}, \quad \forall u \in W_{0}^{1, m^{-}}(\Omega) .
$$

Indeed, assume for a contradiction that relation (11) is not true for each $\epsilon>0$. Then there exists $\epsilon_{0}>0$ and a sequence $\left(v_{n}\right) \subset W_{0}^{1, m^{-}}(\Omega)$ such that $\left|v_{n}\right|_{s}=1$ and

$$
\epsilon_{0}|| \nabla v_{n}||_{m^{-}}+n\left|v_{n}\right|_{m^{-}}<1, \quad \forall n \text {. }
$$

Then it is clear that $\left(v_{n}\right)$ is bounded in $W_{0}^{1, m^{-}}(\Omega)$ and $\left|v_{n}\right|_{m^{-}} \rightarrow 0$. Thus, we deduce that, passing eventually to a subsequence, we can assume that $v_{n}$ converges weakly to a function $v$ in $W_{0}^{1, m^{-}}(\Omega)$ and actually $v=0$. Since $s \in\left(1, \frac{N m^{-}}{N-m^{-}}\right)$, it follows by the Rellich-Kondrachov theorem that $W_{0}^{1, m^{-}}(\Omega)$ is compactly embedded in $L^{s}(\Omega)$ and thus $v_{n}$ converges to 0 in $L^{s}(\Omega)$. On the other hand, since $\left|v_{n}\right|_{s}=1$ for each $n$, we deduce that $|v|_{s}=1$, which is a contradiction. Hence (11) is true.

Next, we note that since $r^{-}>N / m^{-}, m^{-} \cdot r^{-^{\prime}}<N m^{-} /\left(N-m^{-}\right)$, where $r^{-^{\prime}}=r^{-} /\left(r^{-}-1\right)$. Thus, by Hölder's inequality, we have

$$
\int_{\Omega}|V(x)| \cdot|u|^{m^{-}} d x \leq|V|_{r^{-}} \cdot|u|_{m^{-} \cdot r^{-}}^{m^{-}}, \quad \forall u \in W_{0}^{1, m^{-}}(\Omega) .
$$

Combining the last inequality with relation (11), we infer that relation (10) is true.

Arguments similar to those used in the proof of relation (10), combined with the fact that since $r^{-}>N / m^{-}$we also have $r^{-}>N / m^{+}$, imply that there exists $D_{\epsilon}^{\prime \prime}$ satisfying

$$
\begin{aligned}
\int_{\Omega}|V(x)| \cdot|u|^{m^{+}} d x \leq \epsilon \int_{\Omega}|\nabla u|^{m^{+}} d x+D_{\epsilon}^{\prime \prime}|V|_{r^{-}} \int_{\Omega}|u|^{m^{+}} & d x, \\
& \forall u \in W_{0}^{1, m^{+}}(\Omega) .
\end{aligned}
$$

Using relation (2), we deduce that $m^{-} \leq m^{+}<p_{1}(x)$ for any $x \in \bar{\Omega}$ and thus $W_{0}^{1, p_{1}(x)}(\Omega) \subset W_{0}^{1, m^{ \pm}}(\Omega)$. In other words, relations (10) and (12) are true for any 
$u \in X$. Moreover, in the right-hand sides of inequalities (10) and (12) we can take $|V|_{r(x)}$ instead of $|V|_{r^{-}}$since $L^{r(x)}(\Omega)$ is continuously embedded in $L^{r^{-}}(\Omega)$ via inequality (6).

Finally, we point out that since by (2) we have $p_{2}(x)<m^{-} \leq m(x) \leq m^{+}<$ $p_{1}(x)$ for each $x \in \bar{\Omega}$, we deduce that

$$
\left.\left|\int_{\Omega} \frac{V(x)}{m(x)}\right| u\right|^{m(x)} d x\left|\leq \frac{1}{m^{-}} \int_{\Omega}\right| V(x) \mid \cdot\left(|u|^{m^{-}}+|u|^{m^{+}}\right) d x, \quad \forall u \in X
$$

and

$$
\begin{aligned}
\int_{\Omega}\left(|\nabla u|^{m^{-}}\right. & \left.+|\nabla u|^{m^{+}}\right) d x \\
& \leq 2 p_{1}^{+} \cdot \int_{\Omega}\left(\frac{1}{p_{1}(x)}|\nabla u|^{p_{1}(x)} d x+\frac{1}{p_{2}(x)}|\nabla u|^{p_{2}(x)}\right) d x, \quad \forall u \in X .
\end{aligned}
$$

Relations (10), (12), (13) and (14) lead to the result that Lemma 1 is true.

Lemma 2. The following relations are hold:

$$
\lim _{\|u\| \rightarrow \infty} \frac{J_{V}(u)}{I(u)}=\infty
$$

and

$$
\lim _{\|u\| \rightarrow 0} \frac{J_{V}(u)}{I(u)}=\infty .
$$

Proof. First, we note that by (2), $q_{1}(x)<m^{ \pm}<q_{2}(x)$ for any $x \in \bar{\Omega}$. Thus, it is clear that

$$
|u(x)|^{m^{-}}+|u(x)|^{m^{+}} \leq 2\left(|u(x)|^{q_{1}(x)}+|u(x)|^{q_{2}(x)}\right), \quad \forall x \in \bar{\Omega} \text { and } \forall u \in X .
$$

Integrating the above inequality over $\Omega$, we infer that

$$
\frac{\int_{\Omega}\left(|u|^{m^{-}}+|u|^{m^{+}}\right) d x}{\int_{\Omega}\left(|u|^{q_{1}(x)}+|u|^{q_{2}(x)}\right) d x} \leq 2, \quad \forall u \in X .
$$

Using Lemma 1, we find that for $\epsilon \in(0,1)$ there exists $C_{\epsilon}>0$ such that

$$
\frac{J_{V}(u)}{I(u)} \geq \frac{\frac{1-\epsilon}{p_{1}^{+}} \int_{\Omega}\left(|\nabla u|^{p_{1}(x)}+|\nabla u|^{p_{2}(x)}\right) d x-C_{\epsilon}|V|_{r(x)} \int_{\Omega}\left(|u|^{m^{-}}+|u|^{m^{+}}\right) d x}{\frac{1}{q_{2}^{-}} \int_{\Omega}\left(|u|^{q_{1}(x)}+|u|^{q_{2}(x)}\right) d x},
$$

for any $u \in X$.

By the above inequality and relation (17), we deduce that there exist some positive constants $\beta>0$ and $\gamma>0$ such that

$$
\frac{J_{V}(u)}{I(u)} \geq \frac{\beta \int_{\Omega}\left(|\nabla u|^{p_{1}(x)}+|\nabla u|^{p_{2}(x)}\right) d x}{\int_{\Omega}\left(|u|^{q_{1}(x)}+|u|^{q_{2}(x)}\right) d x}-\gamma|V|_{r(x)}, \quad \forall u \in X .
$$


For any $u \in X$ with $\|u\|>1$, relation (18) implies

$$
\frac{J_{V}(u)}{I(u)} \geq \frac{\beta \int_{\Omega}|\nabla u|^{p_{1}(x)} d x}{|u|_{q_{1}^{-}}^{q_{1}^{-}}+|u|_{q_{1}^{+}}^{q_{1}^{+}}+|u|_{q_{2}^{-}}^{q_{2}^{-}}+|u|_{q_{2}^{+}}^{q_{2}^{+}}}-\gamma|V|_{r(x)}, \quad \forall u \in X \text { with }\|u\|>1 .
$$

Now, taking into account the continuous embedding of $X$ in $L^{q_{i}^{ \pm}}(\Omega)$ for $i=1,2$ we deduce the existence of a positive constant $\delta>0$ such that

$$
\frac{J_{V}(u)}{I(u)} \geq \frac{\delta\|u\|_{1}^{p_{1}^{-}}}{\|u\|^{q_{1}^{-}}+\|u\|_{1}^{q_{1}^{+}}+\|u\|_{q_{2}^{-}}+\|u\|^{q_{2}^{+}}}-\gamma|V|_{r(x)}, \quad \forall u \in X \text { with }\|u\|>1 .
$$

Since $p_{1}^{-}>q_{1}^{+} \geq q_{1}^{-} \geq q_{2}^{+} \geq q_{2}^{-}$, passing to the limit as $\|u\| \rightarrow \infty$ in the above inequality we deduce that relation (15) is true.

Next, we remark that since $p_{1}(x)>p_{2}(x)$ for any $x \in \bar{\Omega}$, the space $W_{0}^{1, p_{1}(x)}(\Omega)$ is continuously embedded in $W_{0}^{1, p_{2}(x)}(\Omega)$. Thus, if $\|u\| \rightarrow 0$, then $\|u\|_{1} \rightarrow 0$.

The above remarks enable us to affirm that for any $u \in X$ with $\|u\|<1$ small enough we have $\|u\|_{1}<1$.

On the other hand, since (3) is true, we deduce that $W_{0}^{1, p_{2}(x)}(\Omega)$ is continuously embedded in $L^{q_{i}^{ \pm}}(\Omega)$ with $i=1,2$. It follows that there exist four positive constants $d_{i 1}$ and $d_{i 2}$ with $i=1,2$ such that

$$
\|u\|_{1} \geq d_{i 1} \cdot|u|_{q_{i}^{+}}, \quad \forall u \in W_{0}^{1, p_{2}(x)}(\Omega) \text { and } i=1,2
$$

and

$$
\|u\|_{1} \geq d_{i 2} \cdot|u|_{q_{i}^{-}}, \quad \forall u \in W_{0}^{1, p_{2}(x)}(\Omega) \text { and } i=1,2 .
$$

Thus, for any $u \in X$ with $\|u\|<1$ small enough, relation (18) implies

$$
\frac{J_{V}(u)}{I(u)} \geq \frac{\beta \int_{\Omega}|\nabla u|^{p_{2}(x)} d x}{|u|_{q_{1}^{-}}^{q_{1}^{-}}+|u|_{q_{1}^{+}}^{q_{1}^{+}}+|u|_{q_{2}^{-}}^{q_{2}^{-}}+|u|_{q_{2}^{+}}^{q_{2}^{+}}}-\gamma|V|_{r(x)} .
$$

Next, relations (8), (19) and (20) yield that there exists a constant $\xi>0$ such that

$$
\frac{J_{V}(u)}{I(u)} \geq \frac{\xi\|u\|_{1}^{p_{2}^{+}}}{\|u\|_{1}^{q_{1}^{-}}+\|u\|_{1}^{q_{1}^{+}}+\|u\|_{1}^{q_{2}^{-}}+\|u\|_{1}^{q_{2}^{+}}}-\gamma|V|_{r(x)},
$$

for any $u \in X$ with $\|u\|<1$ small enough. Since $p_{2}^{+}<q_{2}^{-} \leq q_{2}^{+} \leq q_{1}^{-} \leq q_{1}^{+}$, passing to the limit as $\|u\| \rightarrow 0$ (and thus $\|u\|_{1} \rightarrow 0$ ) in the above inequality, we deduce that relation (16) is true. The proof of Lemma 2 is complete.

Remark 1. We point out that by relation (18) and using similar arguments as in the proof of Theorem 1 (Step 1) in [23], we find that for $V$ given and satisfying (4) the quotient $J_{V}(u) / I(u)$ is bounded from below for $u \in X \backslash\{0\}$, i.e., $E(V)$ is a real number. 
Lemma 3. There exists $u \in X \backslash\{0\}$ such that $J_{V}(u) / I(u)=E(V)$.

Proof. Let $\left(u_{n}\right) \subset X \backslash\{0\}$ be a minimizing sequence for $E(V)$, that is,

$$
\lim _{n \rightarrow \infty} \frac{J_{V}\left(u_{n}\right)}{I\left(u_{n}\right)}=E(V) .
$$

By relation (15), it is clear that $\left\{u_{n}\right\}$ is bounded in $X$. Since $X$ is reflexive, it follows that there exists $u \in X$ such that, up to a subsequence, $\left(u_{n}\right)$ converges weakly to $u$ in $X$. On the other hand, arguments similar to those used in the proof of Lemma 3.4 in [21] show that the functional $J_{0}$ (obtained for $V=0$ on $\Omega$ ) is weakly lower semi-continuous. Thus,

$$
\liminf _{n \rightarrow \infty} J_{0}\left(u_{n}\right) \geq J_{0}(u)
$$

By the compact embedding theorem for spaces with variable exponent and assumption (2), it follows that $X$ is compactly embedded in $L^{\sigma(x)}(\Omega)$ (where $\sigma(x)=$ $m(x) \cdot r(x) /(r(x)-1))$ and $L^{q_{i}(x)}(\Omega)$ with $i=1,2$. Thus, $\left(u_{n}\right)$ converges strongly in $L^{\sigma(x)}(\Omega)$ and $L^{q_{i}(x)}(\Omega)$ with $i=1,2$. Then, by relations (6) and (9), it follows that

$$
\lim _{n \rightarrow \infty} I\left(u_{n}\right)=I(u)
$$

and

$$
\lim _{n \rightarrow \infty} \int_{\Omega} V(x)\left|u_{n}\right|^{m(x)} d x=\int_{\Omega} V(x)|u|^{m(x)} d x .
$$

Relations (22), (23) and (24) imply that if $u \neq \equiv 0$, then

$$
\frac{J_{V}(u)}{I(u)}=E(V) \text {. }
$$

Thus, in order to conclude that the lemma is true it suffices to show that $u$ is not trivial. Assume the contrary. Then $u_{n}$ converges weakly to 0 in $X$ and strongly in $L^{s(x)}(\Omega)$ for any $s(x) \in C(\overline{\boldsymbol{\Omega}})$ with $1<s(x)<N p_{1}(x) /\left(N-p_{1}(x)\right)$ on $\overline{\boldsymbol{\Omega}}$. In other words,

$$
\lim _{n \rightarrow \infty}\left(I\left(u_{n}\right)\right)=0
$$

and

$$
\lim _{n \rightarrow \infty} \int_{\Omega} V(x)\left|u_{n}\right|^{m(x)} d x=0 .
$$

If $\epsilon \in(0,|E(V)|)$ is fixed by relation (21), we deduce that for $n$ large enough we have

$$
\left|J_{V}\left(u_{n}\right)-E(V) I\left(u_{n}\right)\right|<\epsilon I\left(u_{n}\right)
$$


or

$$
(|E(V)|-\epsilon) I\left(u_{n}\right)<J_{V}\left(u_{n}\right)<(|E(V)|+\epsilon) I\left(u_{n}\right) .
$$

Passing to the limit in the above inequalities and taking into account that relation (25) is true, we find

$$
\lim _{n \rightarrow \infty} J_{V}\left(u_{n}\right)=0 .
$$

Next, by relation (26), we get

$$
\lim _{n \rightarrow \infty} J_{0}\left(u_{n}\right)=0 .
$$

This fact combined with relation (9) implies that $u_{n}$ actually converges strongly to 0 in $X$, i.e., $\lim _{n \rightarrow \infty}\left\|u_{n}\right\|=0$. This information and relation (16) yield

$$
\lim _{n \rightarrow \infty} \frac{J_{V}\left(u_{n}\right)}{I\left(u_{n}\right)}=\infty,
$$

which is a contradiction. Thus, $u \neq \equiv 0$. The proof of Lemma 3 is complete.

By Lemma 3, we conclude that there exists $u \in X \backslash\{0\}$ such that

$$
\frac{J_{V}(u)}{I(u)}=E(V)=\inf _{w \in X \backslash\{0\}} \frac{J_{V}(w)}{I(w)} .
$$

Then, for any $w \in X$, we have

$$
\left.\frac{d}{d \epsilon} \frac{J_{V}(u+\epsilon w)}{I(u+\epsilon w)}\right|_{\epsilon=0}=0 .
$$

A simple computation yields

$$
\left\langle J_{V}^{\prime}(u), w\right\rangle I(u)-J_{V}(u)\left\langle I^{\prime}(u), w\right\rangle=0,
$$

for all $w \in X$. Relation (28) combined with the fact that $J_{V}(u)=E(V) \cdot I(u)$ and $I(u) \neq 0$ implies that $E(V)$ is an eigenvalue of problem (1).

Next, we show that any $\lambda \in(E(V), \infty)$ is an eigenvalue of problem (1).

Let $\lambda \in(E(V), \infty)$ be arbitrary but fixed. Define $T_{V, \lambda}: X \rightarrow \mathbb{R}$ by

$$
T_{V, \lambda}(u)=J_{V}(u)-\lambda I(u) .
$$

Clearly, $T_{V, \lambda} \in C^{1}(X, \mathbb{R})$ with

$$
\left\langle T_{V, \lambda}^{\prime}(u), v\right\rangle=\left\langle J_{V}^{\prime}(u), v\right\rangle-\lambda\left\langle I^{\prime}(u), v\right\rangle, \quad \forall u \in X .
$$

Thus, $\lambda$ is an eigenvalue of problem (1) if and only if there exists $u_{\lambda} \in X \backslash\{0\}$, a critical point of $T_{V, \lambda}$. 
With similar arguments to the proof of relation (15), we can show that $T_{V, \lambda}$ is coercive, i.e., $\lim _{\|u\| \rightarrow \infty} T_{V, \lambda}(u)=\infty$. On the other hand, as we have already remarked, arguments similar to those used in the proof of Lemma 3.4 in [21] show that the functional $T_{V, \lambda}$ is weakly lower semi-continuous. These two facts enable us to apply Theorem 1.2 in [30] in order to prove that there exists $u_{\lambda} \in X$, a global minimum point of $T_{V, \lambda}$, and thus a critical point of $T_{V, \lambda}$. It is enough to show that $u_{\lambda}$ is not trivial. Indeed, since

$$
E(V)=\inf _{u \in X \backslash\{0\}} \frac{J_{V}(u)}{I(u)} \quad \text { and } \quad \lambda>E(V),
$$

it follows that there exists $v_{\lambda} \in X$ such that

$$
J_{V}\left(v_{\lambda}\right)<\lambda I\left(v_{\lambda}\right),
$$

or

$$
T_{V, \lambda}\left(v_{\lambda}\right)<0 .
$$

Thus

$$
\inf _{X} T_{V, \lambda}<0,
$$

and we conclude that $u_{\lambda}$ is a nontrivial critical point of $T_{V, \lambda}$, or $\lambda$ is an eigenvalue of problem (1).

Finally, we prove that each $\lambda<F(V)$ is not an eigenvalue of problem (1). With that end in view, we assume for a contradiction that there exists an eigenvalue $\lambda<F(V)$ of problem (1). It follows that there exists $u_{\lambda} \in X \backslash\{0\}$ such that

$$
\left\langle J_{V}^{\prime}\left(u_{\lambda}\right), u_{\lambda}\right\rangle=\lambda\left\langle I^{\prime}\left(u_{\lambda}\right), u_{\lambda}\right\rangle .
$$

Since $u_{\lambda} \neq 0$, we have $\left\langle I^{\prime}\left(u_{\lambda}\right), u_{\lambda}\right\rangle>0$. Using this fact and the definition of $F(V)$, we see that

$$
\left\langle J_{V}^{\prime}\left(u_{\lambda}\right), u_{\lambda}\right\rangle=\lambda\left\langle I^{\prime}\left(u_{\lambda}\right), u_{\lambda}\right\rangle<F(V)\left\langle I^{\prime}\left(u_{\lambda}\right), u_{\lambda}\right\rangle \leq\left\langle J_{V}^{\prime}\left(u_{\lambda}\right), u_{\lambda}\right\rangle .
$$

Obviously, this is a contradiction. We deduce that each $\lambda \in(-\infty, F(V))$ is not an eigenvalue of problem (1). Furthermore, it is clear that $E(V) \geq F(V)$.

The proof of Theorem 1 is complete.

Remark 2. We note that in the case when $V=0$ in $\Omega$, the same arguments as in the proof of Theorem 1 (Step 1) in [23] assure that $E(0)>0$. 


\section{Proof of Theorem 2}

Let $S$ be a convex, bounded and closed subset of $L^{r(x)}(\Omega)$ and

$$
E_{\star}:=\inf _{V \in S} E(V) .
$$

Clearly, relation (18) shows that $E_{\star}$ is finite.

On the other hand, let $\left(V_{n}\right) \subset S$ be a minimizing sequence for $E_{\star}$, i.e.,

$$
E\left(V_{n}\right) \rightarrow E_{\star}, \quad \text { as } n \rightarrow \infty
$$

Obviously, $\left(V_{n}\right)$ is a bounded sequence; and thus there exists $V_{\star} \in L^{r(x)}(\Omega)$ such that $V_{n}$ converges weakly to $V_{\star}$ in $L^{r(x)}(\Omega)$. Moreover, since $S$ is convex and closed, it is also weakly closed (see, e.g., Theorem III.7 in Brezis [4]); consequently $V_{\star} \in$ $S$.

Next, we show that $E\left(V_{\star}\right)=E_{\star}$.

Indeed, by Theorem 1 , we deduce that for each positive integer $n$, there exists $u_{n} \in X \backslash\{0\}$ such that

$$
\frac{J_{V_{n}}\left(u_{n}\right)}{I\left(u_{n}\right)}=E\left(V_{n}\right) .
$$

Since $\left(E\left(V_{n}\right)\right)$ is a bounded sequence, and by (18) we have

$$
\frac{J_{V_{n}}\left(u_{n}\right)}{I\left(u_{n}\right)} \geq \beta \frac{J_{0}\left(u_{n}\right)}{I\left(u_{n}\right)}-C \text { for any } n,
$$

where $C$ is a positive constant, we infer that $\left(u_{n}\right)$ is bounded in $X$ and cannot contain a subsequence converging to 0 (otherwise, we obtain a contradiction by applying Lemma 2). Thus, there exists $u_{0} \in X \backslash\{0\}$ such that $\left(u_{n}\right)$ converges weakly to $u_{0}$ in $X$. Using the Rellich-Kondrachov theorem, we deduce that $\left(u_{n}\right)$ converges strongly to $u_{0}$ in $L^{s(x)}(\Omega)$ for any $s(x) \in C(\bar{\Omega})$ satisfying $1<s(x)<$ $N p_{1}(x) /\left(N-p_{1}(x)\right)$ for any $x \in \bar{\Omega}$. In particular, using conditions (2), (3) and (4), we deduce that $\left(u_{n}\right)$ converges to $u_{0}$ in $L^{m(x)}(\Omega)$ and in $L^{m(x) \cdot r^{\prime}(x)}(\Omega)$, where $r^{\prime}(x)=r(x) /(r(x)-1)$. Using this information, inequality (6) and the fact that $V_{\star} \in L^{r(x)}(\Omega)$ and $\left(V_{n}\right)$ is bounded in $L^{r(x)}(\Omega)$, we find that

$$
\lim _{n \rightarrow \infty} \int_{\Omega} \frac{V_{\star}(x)}{m(x)}\left|u_{n}\right|^{m(x)} d x=\int_{\Omega} \frac{V_{\star}(x)}{m(x)}\left|u_{0}\right|^{m(x)} d x
$$

and

$$
\lim _{n \rightarrow \infty} \int_{\Omega}\left(\frac{V_{n}(x)}{m(x)}\left|u_{n}\right|^{m(x)}-\frac{V_{n}(x)}{m(x)}\left|u_{0}\right|^{m(x)}\right) d x=0 .
$$


On the other hand, since $\left(V_{n}\right)$ converges weakly to $V_{\star}$ in $L^{r(x)}(\Omega)$ and $u_{0} \in$ $L^{m(x) \cdot r^{\prime}(x)}(\Omega)$, where $r^{\prime}(x)=r(x) /(r(x)-1)$, we deduce

$$
\lim _{n \rightarrow \infty} \int_{\Omega} \frac{V_{n}(x)}{m(x)}\left|u_{0}\right|^{m(x)} d x=\int_{\Omega} \frac{V_{\star}(x)}{m(x)}\left|u_{0}\right|^{m(x)} d x .
$$

Combining the equality

$$
\begin{aligned}
\int_{\Omega} \frac{V_{\star}(x)}{m(x)}\left|u_{n}\right|^{m(x)} d x-\int_{\Omega} \frac{V_{n}(x)}{m(x)}\left|u_{n}\right|^{m(x)} d x \\
=\int_{\Omega} \frac{V_{\star}(x)}{m(x)}\left|u_{n}\right|^{m(x)} d x-\int_{\Omega} \frac{V_{\star}(x)}{m(x)}\left|u_{0}\right|^{m(x)} d x \\
\quad+\int_{\Omega} \frac{V_{\star}(x)}{m(x)}\left|u_{0}\right|^{m(x)} d x-\int_{\Omega} \frac{V_{n}(x)}{m(x)}\left|u_{0}\right|^{m(x)} d x \\
+\int_{\Omega} \frac{V_{n}(x)}{m(x)}\left|u_{0}\right|^{m(x)} d x-\int_{\Omega} \frac{V_{n}(x)}{m(x)}\left|u_{n}\right|^{m(x)} d x
\end{aligned}
$$

with relations (30), (31) and (32), we obtain

$$
\lim _{n \rightarrow \infty} \int_{\Omega}\left(\frac{V_{\star}(x)}{m(x)}\left|u_{n}\right|^{m(x)}-\frac{V_{n}(x)}{m(x)}\left|u_{n}\right|^{m(x)}\right) d x=0 .
$$

Since

$$
E\left(V_{\star}\right)=\inf _{u \in X \backslash\{0\}} \frac{J_{V_{\star}}(u)}{I(u)},
$$

it follows that

$$
E\left(V_{\star}\right) \leq \frac{J_{V_{\star}}\left(u_{n}\right)}{I\left(u_{n}\right)} .
$$

Combining the above inequality and equality (29) gives

$$
E\left(V_{\star}\right) \leq \frac{J_{V_{\star}}\left(u_{n}\right)-J_{V_{n}}\left(u_{n}\right)}{I\left(u_{n}\right)}+E\left(V_{n}\right) .
$$

Taking into account the result of relation (33), the fact that $I\left(u_{n}\right)$ is bounded and does not converge to 0 and $\left(E\left(V_{n}\right)\right)$ converges to $E_{\star}$, then passing to the limit as $n \rightarrow \infty$ in the last inequality, we infer that

$$
E\left(V_{\star}\right) \leq E_{\star} .
$$

But using the definition of $E_{\star}$ and the fact that $V_{\star} \in S$, we conclude that actually

$$
E\left(V_{\star}\right)=E_{\star} .
$$

The proof of Theorem 2 is complete. 


\section{Proof of Theorem 3}

(a) First, we show that function $E_{\star}$ is not constant. Indeed, by Remark 2, we point out that $E_{\star}(0)=E(0)>0$. On the other hand, by Theorem 1 in [23], it follows that

$$
\lambda_{m}:=\inf _{u \in X \backslash\{0\}} \frac{\int_{\Omega} \frac{1}{p_{1}(x)}|\nabla u|^{p_{1}(x)} d x+\int_{\Omega} \frac{1}{p_{2}(x)}|\nabla u|^{p_{2}(x)} d x}{\int_{\Omega} \frac{1}{m(x)}|u|^{m(x)} d x}>0 .
$$

Moreover, Lemma 2 in [23] implies that there exists $u_{m} \in X \backslash\{0\}$ such that

$$
\lambda_{m}=\frac{\int_{\Omega} \frac{1}{p_{1}(x)}\left|\nabla u_{m}\right|^{p_{1}(x)} d x+\int_{\Omega} \frac{1}{p_{2}(x)}\left|\nabla u_{m}\right|^{p_{2}(x)} d x}{\int_{\Omega} \frac{1}{m(x)}\left|u_{m}\right|^{m(x)} d x} .
$$

Thus, taking $V_{m}(x)=-\lambda_{m}$ for all $x \in \Omega$, we see that $V_{m} \in L^{\infty}(\Omega) \subset L^{r(x)}(\Omega)$ and

$$
\frac{J_{V_{m}}\left(u_{m}\right)}{I\left(u_{m}\right)}=0 \text {. }
$$

It follows that

$$
E\left(V_{m}\right) \leq 0
$$

and we find

$$
E_{\star}\left(\lambda_{m}\right) \leq 0
$$

We conclude that $E_{\star}$ is not constant. Furthermore, we point out that a similar proof to that presented above shows that function $E_{\star}$ also takes negative values. To support that idea simply note that by Theorem 1 in [23], for each $\lambda>\lambda_{m}$ there exists $u_{\lambda} \in X \backslash\{0\}$ such that, taking $V_{\lambda}=-\lambda$ for all $x \in \Omega$, we have

$$
\frac{J_{V_{\lambda}}\left(u_{\lambda}\right)}{I\left(u_{\lambda}\right)}<0
$$

Next, we note that $E_{\star}$ decreases monotonically. Indeed, if we consider $0 \leq R_{1}<R_{2}$, it is clear that $\bar{B}_{R_{1}}(0) \subset \bar{B}_{R_{2}}(0)$. Then the definition of function $E_{\star}$ implies $E_{\star}\left(R_{1}\right) \geq E_{\star}\left(R_{2}\right)$.

(b) Finally, we show that the function $E_{\star}$ is continuous. Let $R>0$ and $t \in$ $(0, R)$ be fixed. We verify that $\lim _{t \unlhd 0} E_{\star}(R+t)=\lim _{t \unlhd 0} E_{\star}(R-t)=E_{\star}(R)$.

First, we prove that $\lim _{t \succ 0} E_{\star}(R+t)=E_{\star}(R)$. By Theorem 3(a), we have

$$
E_{\star}(R) \geq E_{\star}(R+t) .
$$

Moreover, by Theorem 2, it follows that there exists $V_{R+t} \in \bar{B}_{R+t}(0)$ (i.e., $\left|V_{R+t}\right|_{r(x)} \leq$ $R+t$ ) such that

$$
E\left(V_{R+t}\right)=E_{\star}(R+t) .
$$


Taking now $V_{R, t}:=\frac{R}{R+t} V_{R+t}$, we have

$$
\left|V_{R, t}\right|_{r(x)}=\frac{R}{R+t}\left|V_{R+t}\right|_{r(x)} \leq R,
$$

or $V_{R, t} \in \bar{B}_{R}(0)$. Therefore, clearly $E\left(V_{R, t}\right) \geq E_{\star}(R)$.

On the other hand, by Theorem 1 , there exists $u_{t} \in X \backslash\{0\}$ such that

$$
E\left(V_{R+t}\right)=\frac{J_{V_{R+t}}\left(u_{0}\right)}{I\left(u_{0}\right)} .
$$

Combining the above pieces of information, we find

$$
\begin{aligned}
E_{\star}(R+t)=E\left(V_{R+t}\right) & =\frac{J_{V_{R+t}}\left(u_{t}\right)}{I\left(u_{t}\right)} \\
& =\frac{J_{R+t}^{R} \cdot V_{R, t}}{I\left(u_{t}\right)} \\
& =\frac{R+t}{R} \cdot \frac{J_{V_{R, t}}\left(u_{t}\right)}{I\left(u_{t}\right)}-\frac{t}{R} \cdot \frac{J_{0}\left(u_{t}\right)}{I\left(u_{t}\right)} \\
& \geq \frac{R+t}{R} \cdot E_{\star}(R)-\frac{t}{R} \cdot \frac{J_{0}\left(u_{t}\right)}{I\left(u_{t}\right)} .
\end{aligned}
$$

On the other hand, by relation (18), we have that for each $t \in(0, R)$,

$$
\begin{aligned}
E_{\star}(R) \geq E_{\star}(R+t)=E\left(V_{R+t}\right) & =\frac{J_{V_{R+t}}\left(u_{t}\right)}{I\left(u_{t}\right)} \\
& \geq \beta_{1} \cdot \frac{J_{0}\left(u_{t}\right)}{I\left(u_{t}\right)}-\gamma \cdot\left|V_{R+t}\right|_{r(x)} \\
& =\beta_{1} \cdot \frac{J_{0}\left(u_{t}\right)}{I\left(u_{t}\right)}-\gamma \cdot 2 R,
\end{aligned}
$$

where $\beta_{1}>0$ and $\gamma>0$ are real constants.

Combining the last two inequalities, we deduce

$$
E_{\star}(R) \geq E_{\star}(R+t) \geq \frac{R+t}{R} \cdot E_{\star}(R)-\frac{t}{R} \cdot \frac{E_{\star}(R)+\gamma \cdot 2 R}{\beta_{1}},
$$

for each $t \in(0, R)$.

We conclude that

$$
\lim _{t \searrow 0} E_{\star}(R+t)=E_{\star}(R) .
$$

In the following, we argue that $\lim _{t \unlhd 0} E_{\star}(R-t)=E_{\star}(R)$.

Obviously,

$$
E_{\star}(R) \leq E_{\star}(R-t), \quad \forall t \in(0, R) .
$$

By Theorem 2, there exists $V_{R} \in \bar{B}_{R}(0)$ such that

$$
E_{\star}(R)=E\left(V_{R}\right) .
$$


Moreover, by Theorem 1, there exists $u_{0} \in X \backslash\{0\}$ such that

$$
E\left(V_{R}\right)=\frac{J_{V_{R}}\left(u_{0}\right)}{I\left(u_{0}\right)}
$$

Define now

$$
V_{t}:=\frac{R-t}{R} V_{R}, \quad \forall t \in(0, R) .
$$

Clearly, $V_{t} \in \bar{B}_{R-t}(0)$. Thus, it is clear that

$$
\frac{J_{V_{t}}\left(u_{0}\right)}{I\left(u_{0}\right)} \geq E_{\star}(R-t), \quad \forall t \in(0, R) .
$$

Taking into account the above information, we find

$$
\begin{aligned}
E_{\star}(R)=E\left(V_{R}\right)=\frac{J_{V_{R}}\left(u_{0}\right)}{I\left(u_{0}\right)} & =\frac{J_{\frac{R}{R-t} V_{t}}\left(u_{0}\right)}{I\left(u_{0}\right)} \\
& =\frac{J_{V_{t}}\left(u_{0}\right)}{I\left(u_{0}\right)}+\frac{t}{R-t} \cdot \frac{\int_{\Omega} \frac{V_{t}(x)}{m(x)}\left|u_{0}\right|^{m(x)} d x}{I\left(u_{0}\right)} \\
& \geq E_{\star}(R-t)+\frac{t}{R} \cdot \frac{\int_{\Omega} \frac{V_{R}(x)}{m(x)}\left|u_{0}\right|^{m(x)} d x}{I\left(u_{0}\right)}, \quad \forall t \in(0, R) .
\end{aligned}
$$

We infer

$$
\lim _{t \searrow 0} E_{\star}(R-t)=E_{\star}(R) .
$$

It follows that function $E_{\star}$ is continuous. The proof of Theorem 3 is complete.

Remark 3. By Theorem 3(a), we obtain that $E_{\star}$ decreases monotonically. We note that in the particular case when $q_{1}(x)=m(x)=q_{2}(x)=q$ for each $x \in \bar{\Omega}$, where $q>1$ is a real number for which conditions (2), (3) and (4) are fulfilled, the above-quoted result can be improved, in the sense that we can show that, actually, function $E_{\star}$ is strictly decreasing on $[0, \infty)$. Indeed, letting $0 \leq R_{1}<R_{2}$ be given, we deduce by Theorem 2 that there exists $V_{1} \in \bar{B}_{R_{1}}(0)$ such that

$$
E\left(V_{1}\right)=E_{\star}\left(R_{1}\right) .
$$

Then for each real number $t \in\left(0, R_{2}-R_{1}\right)$, we have $V_{1}-t \in \bar{B}_{R_{2}}(0)$, since $\left|V_{1}-t\right|_{r(x)} \leq\left|V_{1}\right|_{r(x)}+t \leq R_{2}$. Next, by Theorem 1 , there exists $u_{1} \in X \backslash\{0\}$ such that

$$
E\left(V_{1}\right)=\frac{J_{V_{1}}\left(u_{1}\right)}{I\left(u_{1}\right)}
$$

Taking into account all the above remarks, we infer

$$
E_{\star}\left(R_{1}\right)-\frac{t}{2}=E\left(V_{1}\right)-\frac{t}{2}=\frac{J_{V_{1}}\left(u_{1}\right)}{I\left(u_{1}\right)}-\frac{t}{2}=\frac{J_{V_{1}-t}\left(u_{1}\right)}{I\left(u_{1}\right)} \geq E\left(V_{1}-t\right) \geq E_{\star}\left(R_{2}\right),
$$


or

$$
E_{\star}\left(R_{1}\right)>E_{\star}\left(R_{2}\right) .
$$

To conclude this remark, we think it is important to highlight the idea that the above proof supports the fact that in the case when we manipulate homogeneous quantities, we obtain better results than in the case when we deal with nonhomogeneous quantities.

Remark 4. We point out that by Theorem 3(b), we deduce that

$$
E_{\star}(R)=\inf _{s \leq R} E_{\star}(s) \text { and } E_{\star}(R)=\sup _{s \geq R} E_{\star}(s) .
$$

Remark 5. We further note that function $E_{\star}$ can be used in order to define a continuous set function on a subset of $L^{r(x)}(\Omega)$. We still denote each closed ball centered in the origin of radius $R$ from $L^{r(x)}(\Omega)$ by $\bar{B}_{R}(0)$, i.e.,

$$
\bar{B}_{R}(0):=\left\{u \in L^{r(x)}(\Omega) ; \quad|u|_{r(x)} \leq R\right\} .
$$

By Theorem 3(b), we deduce that $E_{\star}$ is a continuous function. By the proof of Theorem 3(a), we have $E_{\star}(0)>0$ and there exists $R_{1}>0$ such that $E_{\star}\left(R_{1}\right)<0$. Thus, we infer that there exists $R_{0}>0$ such that $E_{\star}\left(R_{0}\right)=0$.

We define

$$
\Gamma=\left\{\bar{B}_{R}(0) \backslash \bar{B}_{R_{0}}(0) ; R \geq R_{0}\right\} \subset L^{r(x)}(\Omega)
$$

and $\mu: \Gamma \rightarrow[0, \infty)$ by

$$
\mu\left(\bar{B}_{R}(0) \backslash \bar{B}_{R_{0}}(0)\right)=-E_{\star}(R), \quad \forall R \geq R_{0} .
$$

By Theorem 3(a), we find that function $\mu$ has the following properties:

(1) $\mu(\emptyset)=0$.

(2) For each $S_{1}, S_{2} \in \Gamma$ such that $S_{1} \subset S_{2}$, we have $\mu\left(S_{1}\right) \leq \mu\left(S_{2}\right)$.

Thus, $\mu$ is a set function on $\Gamma$. By Theorem 3(b) and Remark 4 , we have that for each $S \subset \Gamma$,

$$
\mu(S)=\sup _{T \subseteq S} \mu(T) \text { and } \mu(S)=\inf _{T \supseteq S} \mu(T) .
$$

We conclude that $\mu$ is a continuous set function on $\Gamma$.

Acknowledgments. V. Rădulescu is very indebted to Professor Haim Brezis for his continuous support over the years. M. Mihăilesco has been fully supported by Grant CNCSIS PD-117/2010 "Probleme neliniare modelate de operatori diferentiali neomogeni” while V. Rădulescu has been fully supported by Grant CNCSIS PCCE-55/2008 "Sisteme diferentiale in analiza neliniara si aplicatii”. 


\section{REFERENCES}

[1] E. Acerbi and G. Mingione, Gradient estimates for the $p(x)$-Laplacean system, J. Reine Angew. Math. 584 (2005), 117-148.

[2] M. S. Ashbaugh and E. M. Harrell, Maximal and minimal eigenvalues and their associated nonlinear equations, J. Math. Phys. 28 (1987), 1770-1786.

[3] J. F. Bonder and L. M. Del Pezzo, An optimization problem for the first eigenvalue of the $p$ Laplacian plus a potential, Commun. Pure Appl. Anal. 5 (2006), 675-690.

[4] H. Brezis, Analyse Fonctionnelle: Théorie, Méthodes et Applications, Masson, Paris, 1992.

[5] Y. Chen, S. Levine, and M. Rao, Variable exponent, linear growth functionals inimage processing, SIAM J. Appl. Math. 66 (2006), 1383-1406.

[6] L. Diening, Theoretical and Numerical Results for Electrorheological Fluids, Ph.D. thesis, University of Frieburg, Germany, 2002.

[7] L. Diening, P. Hästö and A. Nekvinda, Open problems in variable exponent Lebesgue and Sobolev spaces, in FSDONA04 Proceedings (P. Drábek and J. Rákosník, eds.), Milovy, Czech Republic, 2004, pp. 38-58.

[8] D. E. Edmunds, J. Lang, and A. Nekvinda, On $L^{p(x)}$ norms, Proc. Roy. Soc. London, Ser. A, 455 (1999), 219-225.

[9] D. E. Edmunds and J. Rákosník, Density of smooth functions in $W^{k, p(x)}(\Omega)$, Proc. Roy. Soc. London, Ser. A, 437 (1992), 229-236.

[10] D. E. Edmunds and J. Rákosník, Sobolev embedding with variable exponent, Studia Math. 143 (2000), 267-293.

[11] H. Egnell, Extremal properties of the first eigenvalue of a class of elliptic eigenvalue problems, Ann. Sci. Norm. Sup. Pisa 14 (1987), 1-48.

[12] S. Esedoglu and S. Osher, Decomposition of images by the anisotropic Rudin-Osher-Fatemi model, Comm. Pure Appl. Math. 57 (2004), 1609-1626.

[13] X. Fan, Remarks on eigenvalue problems involving the p(x)-Laplacian, J. Math. Anal. Appl., in press.

[14] X. Fan, Q. Zhang and D. Zhao, Eigenvalues of $p(x)$-Laplacian Dirichlet problem, J. Math. Anal. Appl. 302 (2005), 306-317.

[15] T. C. Halsey, Electrorheological fluids, Science 258 (1992), 761-766.

[16] P. Harjulehto, P. Hästö, M. Koskenoja and S. Varonen, Sobolev capacity on the space $W^{1, p(\cdot)}\left(R^{n}\right)$, J. Funct. Spaces Appl. 1 (2003), 17-33.

[17] O. Kováčik and J. Rákosník, On spaces $L^{p(x)}$ and $W^{1, p(x)}$, Czechoslovak Math. J. 41 (1991), 592-618.

[18] A. Kristály, M. Mihăilescu and V. Rădulescu, Two nontrivial solutions for a non-homogeneous Neumann problem: an Orlicz-Sobolev setting, Proc. Roy. Soc. Edinburgh: Sect. A (Mathematics) 139A (2009), 367-379.

[19] M. Mihăilescu, P. Pucci and V. Rădulescu, Nonhomogeneous boundary value problems in anisotropic Sobolev spaces, C. R. Acad. Sci. Paris, Ser. I 345 (2007), 561-566.

[20] M. Mihăilescu, P. Pucci and V. Rădulescu, Eigenvalue problems for anisotropic quasilinear elliptic equations with variable exponent, J. Math. Anal. Appl. 340 (2008), 687-698.

[21] M. Mihăilescu and V. Rădulescu, A multiplicity result for a nonlinear degenerate problem arising in the theory of electrorheological fluids, Proc. Roy. Soc. London, Ser. A 462 (2006), 2625-2641.

[22] M. Mihăilescu and V. Rădulescu, On a nonhomogeneous quasilinear eigenvalue problem in Sobolev spaces with variable exponent, Proc. Amer. Math. Soc. 135 (2007), 2929-2937.

[23] M. Mihăilescu and V. Rădulescu, Continuous spectrum for a class of nonhomogeneous differential operators, Manuscripta Math. 125 (2008), 157-167. 
[24] M. Mihăilescu and V. Rădulescu, Neumann problems associated to nonhomogeneous differential operators in Orlicz-Sobolev spaces, Ann. Inst. Fourier 58 (2008), 2087-2111.

[25] M. Mihăilescu and V. Rădulescu, Spectrum consisting in an unbounded interval for a class of nonhomogeneous differential operators, Bull. London Math. Soc. 40 (2008), 972-984.

[26] M. Mihăilescu and V. Rădulescu, A continuous spectrum for nonhomogeneous differential operators in Orlicz-Sobolev spaces, Math. Scand. 104 (2009), 132-146.

[27] J. Musielak, Orlicz, Spaces and Modular Spaces, Lecture Notes in Mathematics, 1034, SpringerVerlag, Berlin, 1983.

[28] M. Ružička, Electrorheological Fluids: Modeling and Mathematical Theory, Springer-Verlag, Berlin, 2002.

[29] S. Samko and B. Vakulov, Weighted Sobolev theorem with variable exponent for spatial and spherical potential operators, J. Math. Anal. Appl. 310 (2005), 229-246.

[30] M. Struwe, Variational Methods: Applications to Nonlinear Partial Differential Equations and Hamiltonian Systems, Springer, Heidelberg, 1996.

[31] V. Zhikov, Averaging of functionals in the calculus of variations and elasticity, Math. USSR Izv. 29 (1987), 33-66.

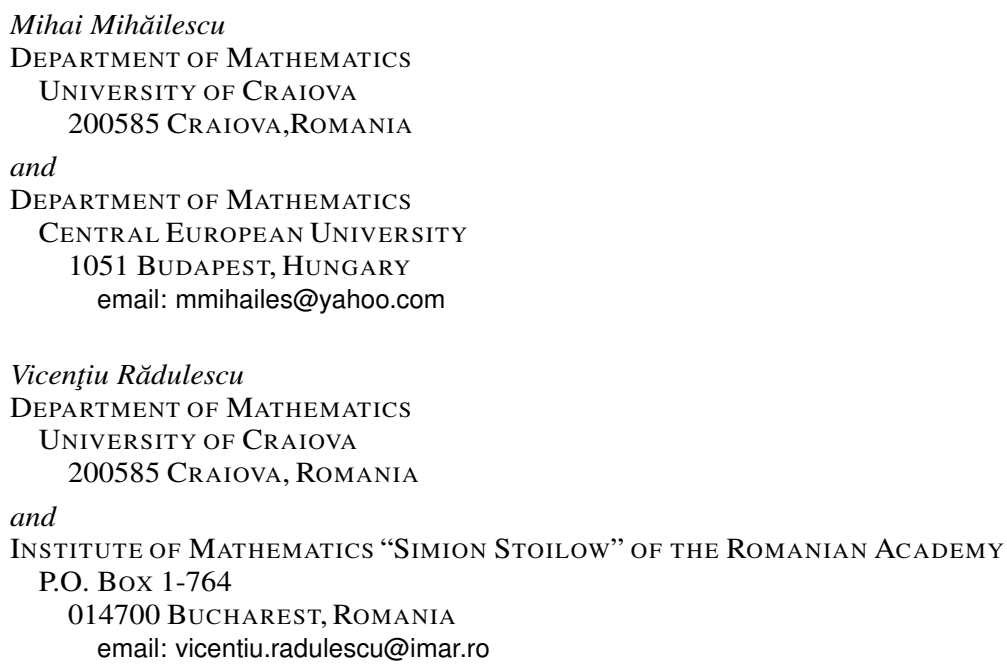

(Received June 8, 2008 and in revised form December 11, 2008) 